\title{
VON RECKLINGHAUSEN'S DISEASE WITH UROGENITAL MANIFESTATION
}

\author{
THIAGO F. NUNES, RENATO P. COSTA, FABIO C. NAVARRO \\ Amaral Carvalho Hospital, Jau, São Paulo, Brazil
}

\begin{abstract}
Neurofibromatosis or Von Recklinghausen's disease is a rare disease that affects the urogenital system in its visceral form. We report on one case where the patient had a recurring mass located in the vesicouterine space that caused irritative urinary symptoms, which was confirmed as uterine cervix neurofibroma following surgery and immunohistochemical study. We stress the importance of a wide resection of such tumors and the need for long-term follow-up due to the high recurrence index and the risk of malignant transformation.
\end{abstract}

Key words: Von Recklinghausen's disease; urogenital system; neoplasm recurrence, local; pelvic neoplasms

Int Braz J Urol. 2005; 31: 153-4

\section{INTRODUCTION}

Neurofibromatosis or Von Recklinghausen's disease is a pathology that features dominant autosomal transmission caused by a mutation in the NF1 gene, which is located on chromosome 17q11.2. It demonstrates $100 \%$ penetration but a highly variable phenotypic expression (1). The incidence of systemic disease is 1 in every 3000 births; however, urogenital involvement is rare, and only about 50 cases with urinary manifestation have been described, in most cases featuring involvement of the bladder (2). It is known that there is a 12 to $29 \%$ (3) risk of malignant degeneration and so follow-up with such patients is important, as is resection of the lesion whenever there is a suspicion of malignant transformation.

\section{CASE REPORT}

A 21-year old Caucasian woman previously diagnosed with Von Recklinghausen's disease type I who had undergone 2 previous operations (exeresis of neurofibroma in her left forearm and retrovesical neurofibroma with bladder preservation 13 years earlier). She was assymptomatic and had undergone abdominal and pelvic computerized tomography (CT) every 6 months during the first 2 years and subsequently every 3 years. Since 2001 she had had irritative urinary symptoms (dysuria, pollakiuria) due to urinary tract infections (on average every 2 months) confirmed by urine culture. Physical examination was normal except for the presence of "café au lait" spots on her entire body.

An abdominal and pelvic CT was then repeated which revealed a homogeneous pelvic mass in the vesicouterine space causing compression of the uterus and ureters and promoting thickening of the bladder wall (Figure-1). Excretory urography showed dilation in the distal third of the left ureter with a sharp tapering in the intramural region. Cystoscopy revealed a large retrotrigonal bulging and the presence of trigonitis. The patient underwent an exploratory laparotomy through an infra-umbilical median incision. During the intra-operative dissection, we 


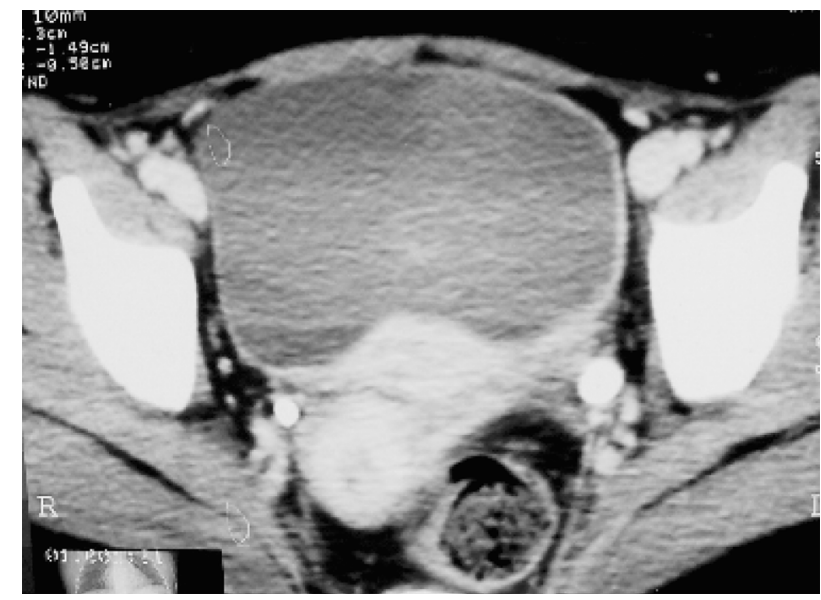

Figure 1 - Computerized abdominal tomography showing a pelvic mass with indefinite limits between the uterus and bladder, causing a bulging in the trigone region.

observed that the retrovesical mass showed a cleavage plane from the bladder which was affecting the uterus. We subsequently performed a total hysterectomy with a wide lesion re-section.

The pathological study through immunohistochemical analysis with the streptavidin-biotin-peroxidase technique evidenced positivity for protein S100 and vimentin antibodies, confirming that the lesion was a diffuse neurofibroma of the uterine cervix (Figure-2).

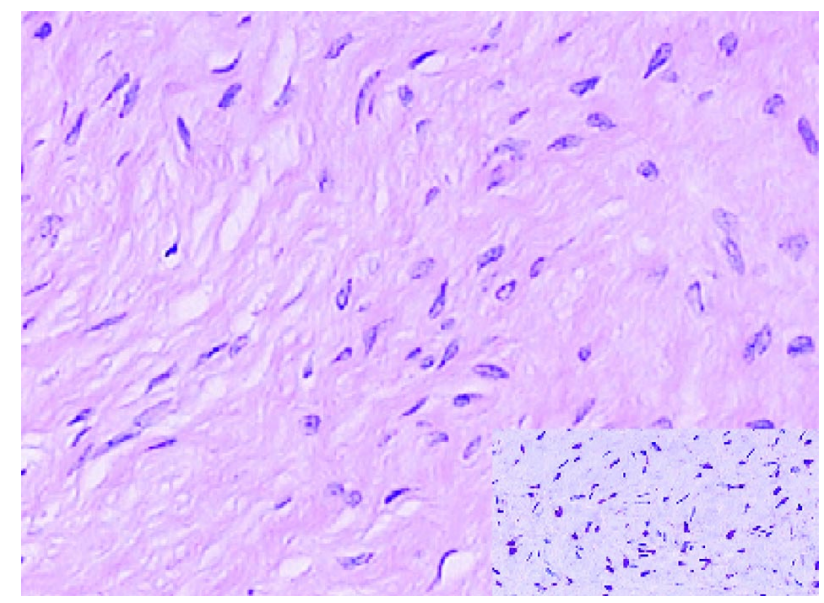

Figure 2 - Histological study showing spindle cells with fibrillar cytoplasm and elongated nuclei characterizing diffuse neurofibroma (HE, X20). Insertion showing nuclear immunoreactivity to protein $S 100$.

\section{COMMENTS}

Neurofibromatosis is a hamartomatous disease of the cell components originating from the neural crest. Neurofibromas are tumors of the autonomic peripheral nerves (Schwann cells) and rarely involve organs in the urogenital system. They most commonly affect the bladder due to the presence of a rich autonomic plexus. The pathogenesis is unknown, however it is known that there is a considerable risk of malignant degeneration (from 12 to $29 \%$ ) which warrants clinical follow-up and serial imaging exams along with tumor exeresis if the mass increases suddenly, since this could suggest malignant transformation. Local recurrence is common, especially in large skin neurofibromas, thus clinical follow-up with CT is required every 6 months during the first 2 years and then annually.

\section{REFERENCES}

1. Brasfield RD, Das Gupta TK: Von Recklinghausen's disease: a clinicopathological study. Ann Surg. 1972; 175: 174-83.

2. Blum MD, Bahnson RR, Carter MF: Urologic manifestations of Von Recklinghausen neurofibromatosis. Urology. 1985; 26: 209-17.

3. Pycha A, Klingler CH, Reiter WJ, Schroth B, Haitel A, Latal D: Von Recklinghausen neurofibromatosis with urinary bladder involvement. Urology. 2001; 58: 106.

Received: October 23, 2004

Accepted after revision: January 21, 2005 